\title{
Contribution of Additive Manufacturing Systems to Supply Chain
}

\author{
Satya Shah \\ Applied Engineering Management \\ Faculty of Engineering \& Science, University of Greenwich \\ Chatham, United Kingdom \\ s.shah@gre.ac.uk \\ Elmira Naghi Ganji \\ Applied Engineering Management \\ Faculty of Engineering \& Science, University of Greenwich \\ Chatham, United Kingdom \\ e.naghiganji@gre.ac.uk
}

\author{
Stefano Mattiuzza \\ Applied Engineering and Management \\ Faculty of Engineering \& Science, University of Greenwich \\ Chatham, United Kingdom \\ s.mattiuzza@gre.ac.uk \\ Alec Coutroubis \\ Applied Engineering and Management \\ Faculty of Engineering \& Science, University of Greenwich \\ Chatham, United Kingdom \\ a.d.coutroubis@gre.ac.uk
}

\begin{abstract}
The current economic pressures have been challenging manufacturing SMEs that play a complicated role in the Supply Chain (SC); and innovation in the manufacturing supply chain which requires new approaches and technologies. Additive Manufacturing (AM) is considered by research studies as pioneering and disruptive technology that can drive such a transformation. This paper is aimed at investigating in what measures can additive manufacturing techniques adopted as an opportunity for the supply chain in SMEs. Initially, the literature review looks into needs and challenges of SMEs in the SC identifying solutions and strategies. The research further analyses AM's impact on the supply chain, evaluating benefits and drawbacks that AM delivers in this context. IT aims to examine the use of strategic maps, analytical tools and suitable tactical paths to implement and integrate this technology within supply chain. The paper describes the potential effects of the open sources networks and hence argues that a gradual integration of AM with traditional technologies is a plausible way for small manufacturers. However, there are several innovative approaches, and this scenario is rapidly evolving. Material and machines costs reduction could deliver significant changes. Therefore, further and updated investigations from a supply chain perspective are recommended, especially looking at practical cases and examples for this study within this context.
\end{abstract}

Keywords - additive manufacturing; supply chain management; manufacturing; innovation and disruptive technolgy.

\section{INTRODUCTION}

In the last decade small and medium manufacturing enterprises (SMEs) have been facing one of the most disruptive and severe periods of economic challenges. The huge growth of Internet, communication technologies and in general the phenomena of globalisation have reduced timing and distances challenging all the existent businesses. The modern day communication is getting smaller; everyone and every place is easily reachable, hence new markets and opportunities, new possibilities of outsourcing, uploaded ways to order or ship products and services are required. At the same time, it means that new competitors at global markets are emerging. In this scenario, a smart supply chain management (SCM) plays a determinant role. This research study aims at investigating how the recent and disruptive technology of AM can drive a supply chain transformation to bring innovation and reduce time-tomarket in the context of manufacturing SMEs. In order to face today's increasingly globalised and dynamic economy, innovation in processes, and supply chain risk management have become essential tools to achieve a competitive advantage.

According to some experts this disruptive technology, which is rapidly continuing to grow, will contribute to carry on a further new industrial revolution related to the digital manufacturing. Generally, AM has always been observed from a technological point of view. People expected that its technological improvement could solve and provide the platform for every production problem. Nevertheless, the high value of this wide invention must be investigated with a horizontal approach, considering the significant impact on the development of a new business model and framework; and most of all its implications towards the wider supply chain. This technology can redesign the supply chain, reducing costs and most of all improving companies' responsiveness.

\section{LITERATURE REVIEW}

This literature review aims to develop a better understanding of the concepts that seem to play a vital role towards the adoption of additive manufacturing methods in manufacturing SMEs. Current literatures will provide relevant materials related to the specific research objectives. The review of this study will be split into several sections and will be structured as follows; the adoption of supply chain management, the key objectives of manufacturing supply chain, the use of additive manufacturing, and the further implications and strategies towards the adoption of AM methods within the supply chain operations. It will also cover the gaps and what the research study intends to address. This study will provide a framework that serves for the future research and analysis of application and use of innovative manufacturing practices within supply chain for SMEs. 


\section{A. Adoption of Supply Chain Management (SCM)}

The aim of every supply chain is to maximize the supply chain surplus which corresponds to the difference between the customer value and the supply chain cost. In other words, working on costs reduction and added value, the final objective is to increase the difference between the revenue obtained from the customer and the overall costs across the supply chain. Moreover, SMEs play a significant role on enterprise scenes and market economies, as they cover about $90 \%$ of all global businesses, with the high number of suppliers through the supply chain. Not surprisingly, they contribute to $70 \%$ of the environmental pollution within the manufacturing sector [1]. Therefore, executing SCM practices, SMEs can achieve significant benefits. Firstly, concerning operations management (reducing inventory, time to market, costs), and then in terms of planning and strategic actions (planning resources, increasing level of service, forecasting demand and reducing risk uncertainty). These types of firms is characterised by limited capacities in personnel and management dimensions, and by weaker cash flow. Researchers organised the main drawbacks and advantages for SMEs successfully. Beyond insufficient financial means, small companies have restricted knowledge of upgraded information technology. Indeed, they frequently rely on obsolete IT tools such as legacy systems. Furthermore, they are strongly influenced by their customers and sometimes not able to manage external pressures such as changes in economic, governmental, socio-cultural \& technological [2].

They have some advantages due to their simplified and lean structure that makes them more agile in their internal operations. Indeed, they can initiate and implement innovations faster than monolithic companies. In fact, they can take advantage of these features, of their good relationship with their employees, to be more flexible and to react faster to market turbulence. Additionally, the owner is usually the developer of the SCM strategic map. This situation facilitates decision making and SCM implementation. Moreover, some studies show that SMEs are very efficient and productive in R\&D. For instance, they innovate more than twice per employee in comparison to large-scale enterprises, and they are more inclined to adopt new and untested technologies [2]. We argue that it is valid just in case of excellences, additionally the fundamental role of a closer relationship with customers. The small dimension of these companies and the direct contact between owner and customers allow improving the quality of the service, to carry on a strategic alliance.

An interesting analysis on SCM between SMEs and largescale enterprises is made by [3]. Their research examined three different hypotheses given a survey carried out within the automotive sector in Germany. Firstly, they confirmed that SMEs regardless of their SC are slightly more vulnerable. Indeed, they are more dependent by original equipment manufacturer (OEM) and they have limited financial liquidity. The second hypothesis is related to the stronger influence of developments towards complexity and effectiveness on SMEs. However, they recognized the limits of their research, due to the unique context of the investigation. Finally, it was demonstrated that both large-scale companies and small companies make significant efforts to manage risk in SC, but implementing different instruments of SCM would be more suitable for them. The former case focuses towards preventing instruments such as improving on-time deliveries, tracking and tracing, demand forecasting, eliminating waste and sources of errors according to lean principles. The latter, on the other hand, acts on supply chain risks by building up redundancies through reactive instruments. They rely on safety stocks, overcapacity production, multiple sourcing, and back -up supplier.

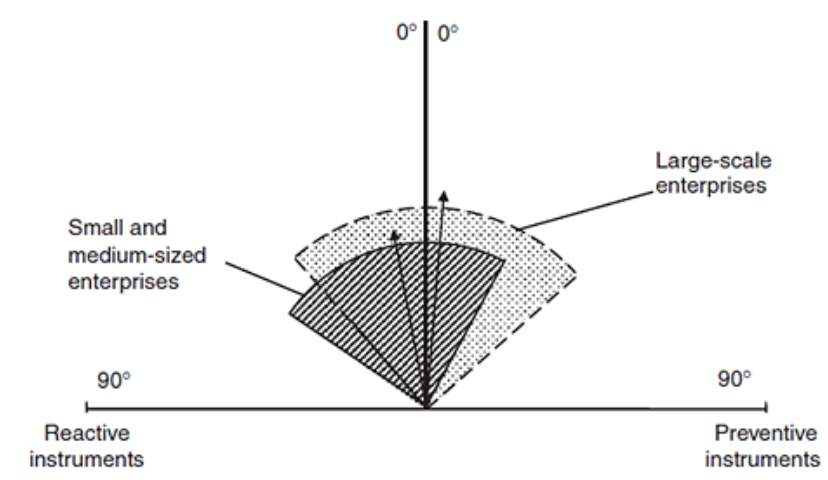

Fig. 1. Different Types of Enterprises. [3]

Overall, as shown in Figure 1 [3], in a range between 0' and 90', these kinds of enterprises are quantitatively similar; however, here is a difference in the selection of their supply chain drivers.

\section{B. Manufacturing Supply Chain Objectives}

Manufacturing firms are increasingly forced to deal with risk issues in their supply chain. In the previous paragraph it was realized that, although their structural limits, SMEs have still the right tools to face risk issues in the supply chain. Nonetheless, to adopt the best solution they need to develop the right strategy and achieve the strategic fit remains the key focus. In other words, once defined the most suitable competitive advantage for the firm, managers must develop the most efficient solution to achieve it. In the manufacturing context, it means integrating the development chain with the logistic chain, to build the most effective supply chain.

Firstly, to improve the right strategy, companies should individuate what product types are being manufactured. Innovative products usually are characterized by unpredictable demand and hence, they require high responsiveness and service level. The firm will focus on meeting every customer needs, minimizing lead time and becoming very responsive. On the contrary, functional products are typified by opposite features; and the product demand is often constant or easily predictable. Consequently, companies can work on costs reduction and therefore providing lower prices. Based on this classification one particular research study smartly sums up the key SCM strategies. Ordinary and functional products require efficient and lean supply chain. It is centred on eliminating waste, executing low cost SC operations, with the purpose of delivering a lower product price to the customer. In particular the most relevant types of waste to be eliminated are: overproduction, waiting time, unnecessary transportation, incorrect processing, excess inventory, unnecessary movement, defects and unused employee creativity [4]. In contrast, in order to gain significant revenues while producing innovative products, companies should adopt the agile supply chain 
practices more frequently. Its main characteristic is the ability to respond to variation in the demand. It should ideally be constituted on modularization so as to provide shorter lead times, flexibility and mass customization. However, in the current dynamic and evolving environments, companies often need to improve both these aspects. Hence, the needs to develop a bride type SC, called leagile supply chain [4]. This model tries to balance cost efficiency and responsiveness through the use of the order de-coupling point (CODP). It works organizing the architecture of SC in modules, then separating the part where production is driven by forecast and the part where is driven by customer orders as shown in Figure 2. The four CODP elements are defined as engineer-to-order (ETO), make-to-order (MTO), assemble-to-order (ATO), and make-to-stock (MTS). P and D refer to the production lead-time and the delivery lead-time respectively [5].

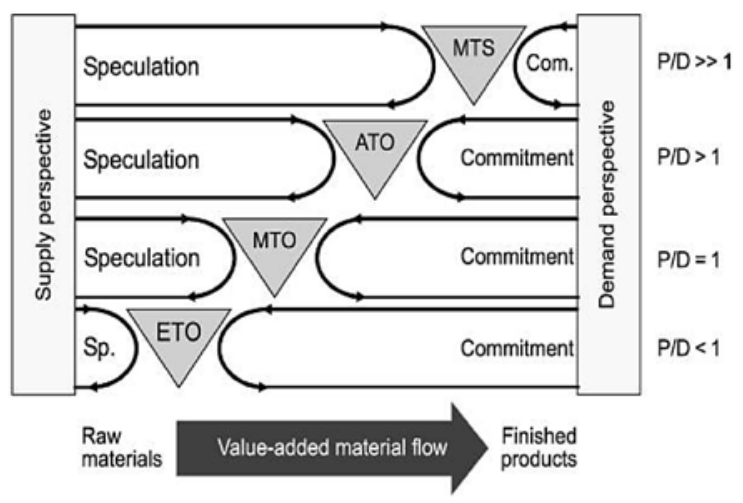

Fig. 2. Generic customer order - decoupling Points [5].

It is a strict analysis and needs the application of the Push/Pull logic. This approach is perfect for SMEs, that needs to be responsive and flexible because of its position in the SC, but at the same time must be competitive in costs.

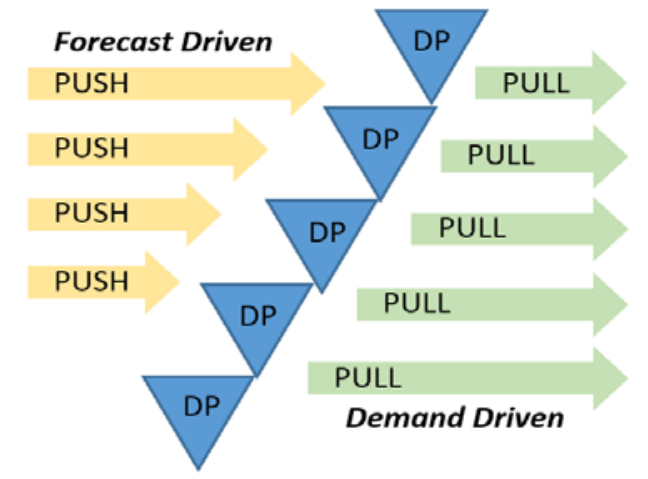

Fig. 3. Customer - demand/forecast driven and push pull strategies (adopted from [5]).

On the basis of the previous observations, firms should individuate the right decoupling point for them, in order to develop a tailored supply chain as shown in Figure 3, where DP refers to demand planning. According to the literature, it is evident from Figure 3 that the effective demand driven chain attempts to create pull for new products instead of pushing the supply into the market from the traditional perspective of the supply chain [6]. In this manufacturing context, where time-tomarket and cost efficiency play a conflicting, but determinant role, additive manufacturing can deliver some important innovations in the technological process. Besides, the supply chain is the part that can take the most advantages of this advanced tool.

\section{Additive Manufacturing Impact on Supply Chain Integration}

Additive manufacturing, also recognized as direct manufacturing, is a recent technology utilizing a threedimensional computer aided design (CAD) file [7], which was developed since the early 80s and has been growing to achieve optimal results in the production of prototyping part initially, and end-user parts in the later years. Polymers and Metal are mostly used in 3D printers, however, new materials like latter, glass and concrete are finding applications nowadays. The main feature of $\mathrm{AM}$ is that objects are produced adding layers of material instead of subtracting it (as the traditional manufacturing machines). It delivers new freedom of design, allowing the direct production of shapes and items that otherwise should have been carried out with two or more manufacturing steps. This relates that the supply chain can be considerably shortened, with reduction of inventory and more flexibility. Nowadays, new market trends are towards mass customization, innovation and responsiveness, and additive manufacturing methods could respond to these necessities more appropriately. Similar research studies sums up in ten points the key advantages of additive manufacturing and this paper investigates most of them reviewed from an SC perspective [8].

In this field, AM has being used for almost 30 years and nowadays, many companies are adopting this technology due to its several advantages. Firstly, a strong prototyping time reduction, means that designing times are reduced and so the firm becomes more responsiveness. This is very important in the designing/contracting phase, especially in the SMEs context, because AM can allow to gain a "first-move advantage", capturing the demand more quickly than competitors. Secondly, it allows to eliminate every error, uncertainty and misunderstanding related to the interpretation of the project, and also to improve the quality of the product thanks to an upgraded prototype. Finally, it delivers an added value in terms of marketing, customer relationship and brand image. The most relevant implication in the production sector is that manufacturing complexity is free and it is possible to reengineer shapes, products and processes thinking additive. In SC terms the main deliver of this aspect consists of lead time reduction and more responsiveness. Studies have also been providing insight on the use of AM technology and the impact on different aspects of supply chain, including suppliers, manufacturing firms, and customers. It has also been argued that AM technologies provide the wider benefits of integrating any additional functionalities within the products during the manufacturing stages and towards optimizing those products for the specific functions. This in turn reduces the amount of sub-production required and towards improving the supplier connection within the network [9]. Research also suggests the lack of any systematic studies carried out the effects of AM technology and its usage on production of customised products within the supply chain. 
In the case of particular products (low volumes, mass customization) it can be hypothesized a dramatic redesign of the supply chain in terms of total decentralization due to the adoption of additive manufacturing. This proves that the company can adopt a distributed production model rather than the traditional and often cost-effective centralized one. The goods are manufactured directly at or near the point of use. Some experts imagine a future scenario where customers can even fabricate goods at home, because everyone will have a 3D printer available for personal use. Some companies have been considering the disruptive logistics change proactively. Research also suggests additive manufacturing using different technologies allowing the creation of items through the addition of successive layers of material. The innovation of 3D printing methods can be used in prototyping or through direct production process for semi or finishes goods [10]. On the other hand, it can be observed the phenomena of re-shoring, because the development of the AM could reduce the need of delocalizing to low-cost developing countries. In fact, additive manufacturing is very low labour intensity, raw materials have the same price everywhere globally and it concerns with less environmental issues. Consequences towards pollution are an aspect analysed also by a similar study [4]. They reviewed the implications of additive manufacturing in the context of SC through the four following principles (1) Green operations, (2) A cost-efficient flexibility, (3) How, where and who being redefined; and (4) Negligible economies of Scale. We have already discussed the first three, and the environmental theme will be readdressed later within the paper. However, additive manufacturing is still not cost-effective for large volumes of goods, and perhaps it will never be fitted for certain types of products. It means, that this technology needs to be examined with a horizontal approach: as a way to integrate and corroborate the traditional manufacturing processes: a complement rather than a substitute.

\section{Further Implications towards the Adoption of AM Methods Within the Supply Chain}

Transforming the manufacturing characteristics from traditional approaches [11], digital manufacturing-driven alterations of supply chain are still being considered as a potential infrastructure technology which needs to emerge incrementally [12]. Moreover, for the complex products there is still a need to study the cross-disciplinary collaboration [12]. Additive Manufacturing is one of the main actors of the new industrial revolution, which is already in progress. It is called Industry 4.0 and consists of digital manufacturing, mass customization, and knowledge intensive production lines. In the aforementioned scenario, the most significant impact is not merely the technology, but the new maker culture. Researchers have faced this fundamental aspect which is related to the value of external knowledge, collaboration, information sharing. Enterprises will still rely on Universities and company labs, but mostly they are forming open sources collaborative and workshops. This interconnected community made of small and large businesses, user groups, consumers, online retailers and social platforms is actually a self-organizing global supply chain [11]. Advanced companies aim to be prepared to manage this new complexity and technology, and so they could take advantage of the combination of digital manufacturing and supply chain, decreasing the SC complexity through more effective solutions. Moreover, identifying the novel integration between digital manufacturing, logistics and equipment use can influence on the relationships among the logistics service providers (LSPs), manufacturers and users [12]. This collaborative environment and the sharing of knowledge aim to expand the strategic scope, maximizing the supply chain surplus. From a practical view it means that in the fabric of the future $R \& D$ and manufacturing are merging. Manufacturers must be aware that the future is toward a hybrid product line that blends complementary mass production and mass customized objects [13]. Among the several numerical methods elaborated to manage inventory, deliveries and in general SC issues, the research aims to present briefly some patterns that were addressed to the AM context. Researchers developed a stochastic optimization model with the aim of providing an analytical tool which allows manufacturers to take the best decision for their supply chain regarding the use of AM rather than traditional manufacturing [14].

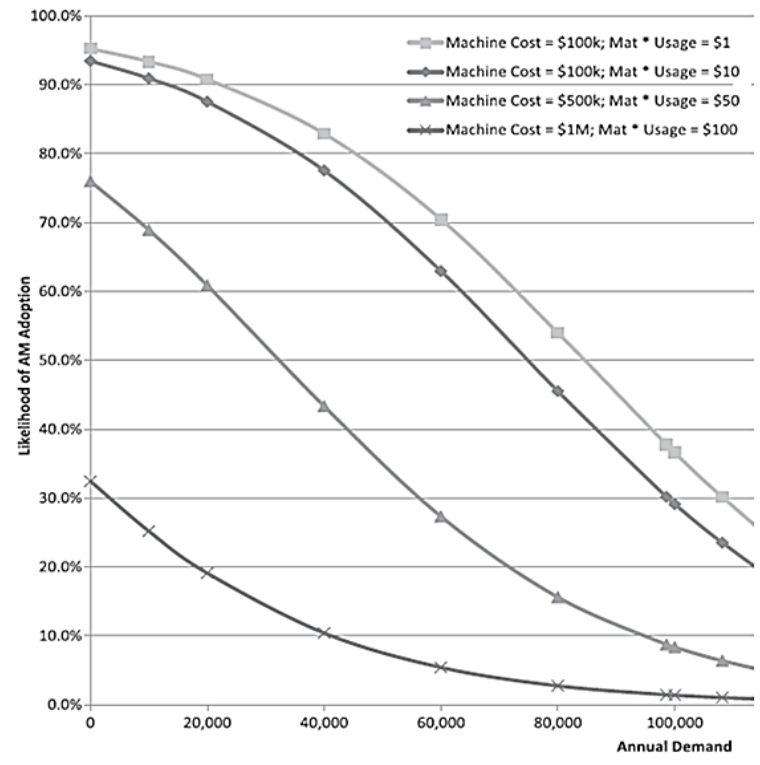

Fig. 4. Likelihood of AM implementation [14].

Using a Stochastic Programme based on eight key parameters they created a model to evaluate the total cost of the SC for traditional and AM production systems. Hence, they conceived a logistic regression model to evaluate the most important factors in order to optimize the results. They found out that the three primary drivers of AM operations are the cost of material, the manufacturing time and the purchase price of the machine. Those factors, in turn, vary according to the annual demand, as shown in Figure 4. This study individuates precisely the most critical factors of this technology. Indeed, if the prices of machinery and raw materials dropped it would be a strong incentive to adopt AM, especially for SMEs that do not have large liquidity. Hence, we cite the recent work of [15] which presents a Joint Economic Lot Size (JELS) analytic model aimed at capturing the impact of AM within the supply chain in a single vendor-single buyer scenario, to find a more profitable joint production and inventory policy. In this model, the 
variables used to optimize the total cost function are the number of shipments and lot size. The impact of additive manufacturing also depends on the nature of industry and sector, however AM is largely been considered as incremental innovation for one, whereas radical for another. Regardless, researchers suggests the development of AM not only to impact certain industries but have large societal importance as whole [16].

\section{RESEARCH METHODOLOGY}

As part of the future aspect of this research study, a combination of research methods and approaches will be adopted within this research. This will include further use of critical literature review analysis, including case study based method investigating manufacturing suppliers, through personal interviews with manufacturing companies. The literature review conducted extensively at the initial stages of the research identified the research gaps in knowledge within this area. Methodology deals with the basic principal question of how to collect analyse and interpret data in a structured manner. The research started from theory and then moved into data, collecting qualitative information from case studies by using questionnaires and interview. The variable for this study was generated from the literature and the relationship to each other is seen within the framework. The deductive approach will also include a qualitative research method that is used to gain more insight. There is numerous research strategies used for attaining answers in any research objectives. However, due to the nature and objective of this study, the use of semistructured questionnaire to clarify the assumption gained from the literature is planned for future research.

\section{CONCLUSIONS AND FUTURE RESEARCH}

The most important features of additive manufacturing innovative technology has been examined. Usually, researchers investigate this topic with a vertical approach, very focused on technological aspects. However in this research study, the authors focused on supply chain perspective. This study revealed that some of the most disruptive transformations come from this tactical and strategical approach. In particular, it concentrated on SMEs, discovering how the technology has all the potential characteristics to drive this kind of firms towards innovation and competitive advantage. Actually, it has already been implemented by many companies, especially regarding rapid prototyping and tooling, or in the case of very small niche products. This research study explored various other paths and opportunities that AM offers, including redesigning the supply chain and the business model entirely, or collaborating in an open source network. However, there are still some practical problems for small and medium manufacturer's regarding machinery and raw material costs; besides, the uncertainty of the market and this new environment, the lack of financial and management means often prevent companies from operating a conversion toward this technology. Hence, the argument towards gradual and hybrid model of integration that enables the blending of complementary additive manufacturing with traditional manufacturing processes is more suitable for SMEs apart from certain cases and products. This paper recommends towards undertaking further studies and researches with the scope of evaluating newer and original paths of innovation that companies are carrying out towards AM implementation. The future focus should aim towards identifying the practical cases within SMEs in order to facilitate the transformation of strategic supply chain management. Moreover, observing and analysing how the overall context is evolving, researchers could investigate the scenario and the implications that likely would descend from decreasing prices and improving current technology infrastructure. On this basis, it could be possible to develop a more detailed and precise analytical model.

\section{REFERENCES}

[1] S. Shah, E. Ganji, S. Hasan, S. "Environmental management systems and sustainability in SMEs," MATEC Web of Conferences, vol. 76, pp. 02006, 2016.

[2] T.A. Chin, A. B. A. Hamid, A. Rasli, and R. Baharun. "Adoption of supply chain management in SMEs," Procedia-Social Behavioral Sciences, vol. 65, pp. 614-619, 2012.

[3] J. H. Thun, M. Drüke, and D. Hoenig. "Managing uncertainty - an empirical analysis of supply chain risk management in small and mediumsize entreprises,” International Journal of Production Research, vol. 49, pp. 5511-5525, 2011.

[4] H. J. Nyman, P. and Sarlin, "From bits to atoms: 3D printing in the context of supply chain strategies," System Sciences, (HICSS), 47th Hawaii International Conference on, pp. 4190-4199, 2014.

[5] J. Wikner, and M. Rudberg, "Integrating production and engineering perspectives on the customer order decoupling point”, International Journal of Operations and Production Management, vol. 25, pp. 623-641, 2005.

[6] S. Emmett, and B. Crocker, The relationship-driven supply chain creating a culture of collaboration throughout the chain. Aldershot, England: Gower Publishing, 2006.

[7] S.H. Khajavi, J. Partanen, J. Holmström, “Additive manufacturing in the spare parts supply chain,” Computers in Industry, vol. 65, pp. 50-63, 2014.

[8] H. Lipson, M. Kurman, Fabricated: The New World of 3 Printing, United States: John Wiley \& Sons, 2013.

[9] K. Oettmeier, and E. Hofmann, "Impact of additive manufacturing technology adoption on supply chain management processes and components”, Journal of Manufacturing Technology Management, vol. 27, pp. 944-968, 2016.

[10] A. Caputo, G. Marzi, and M.M. Pellegrini,” The Internet of Things in manufacturing innovation processes Development and application of a conceptual framework ,” Business Process Management Journal, vol. 22, pp. 383-402, 2016.

[11] T. Igoe, C. Mota. A Strategist's Guide to Digital Fabrication, Strategy Business Publication, vol. 64, 2011.

[12] J. Holmström, J. Partanen, "Digital manufacturing-driven transformations of service supply chains for complex productsk," Supply Chain Managemenent, An International Journal. vol. 19, pp. 421-430, 2014.

[13] M. Despeisse, S. Ford. "The Role of Additive Manufacturing in Improving Resource Efficiency and Sustainability,” IFIP, APMS 2015, pp. 129-136, 2015.

[14] A. Scott, and T.P. Harrison, "Additive Manufacturing in an End-to-End Supply Chain Setting. 3D Printing and Additive Manufacturing,” vol. 2, pp. 65-77, 2015.

[15] A. Milad, S. Zanoni, A. Bacchetti, M. Zanardini, and M. Perona. "Additive Manufacturing Impacts on Production and Logistics Systems," IFAC Conference, vol. 49, pp. 1679-1684, 2016.

[16] H. Steenhuis, and L. Pretorius, "The additive manufacturing innovation: a range of implications,” Journal of Manufacturing Technology Management , vol. 28, pp-xxx, 2017 